(C2007 IEEE. Personal use of this material is permitted. However, permission to reprint/republish this material for advertising or promotional purposes or for creating new collective works for resale or redistribution to servers or lists, or to reuse any copyrighted component of this work in other works must be obtained from the IEEE. 


\section{FRM-Based FIR Filters with Minimum Coefficient Sensitivities}

\author{
Y. C. Lim and Y. J. Yu \\ School of EEE, \\ Nanyang Technological University., \\ Singapore.
}

\author{
K. L. Teo \\ Dept. of Mathematics \& Statistics, \\ Curtin University of Technology, \\ Australia.
}

\author{
T. Saramäki \\ Institute of Signal Processing, Tampere \\ University of Technology, \\ Finland.
}

\begin{abstract}
A method for optimizing FRM-based FIR filters with optimum coefficient sensitivity is presented. This technique can be used in conjunction with nonlinear optimization techniques to design very sharp filters that do not only have very sparse coefficient values but also very low coefficient sensitivity.
\end{abstract}

\section{INTRODUCTION}

$\mathrm{T}$ HE frequency response masking (FRM) technique [120] has received much attention for the synthesis of very sharp digital filters with very sparse coefficients. It has found applications in diverse fields including the synthesis of various types of filters such as half-band filters [21]-[23], 2D filters [24], IIR filters [25]-[28], filter banks [29]-[34], decimators and interpolators [35], [36], and Hilbert transformers [37], [38], FPGA implementations [39-41], transmultiplexer design [42], ECG signal processing [43], hearing aids [44], digital audio [45]-[49] application and analysis, speech recognition [50], array beamforming [51], software radio [52], and noise thermometer [53].

Fig. 1 shows the structure of an FIR filter synthesized using the FRM technique. A filter with z-transform transfer function $H(z)$ is synthesized using a network of sub-filters $H_{a}\left(z^{M}\right), H_{M a}(z), H_{M c}(z)$, and $z^{-\bullet}$ where $z^{-\bullet}$ represents an appropriate negative integer power of $z$ and $M$ is an integer [1]; all the sub-filters have low arithmetic complexities.

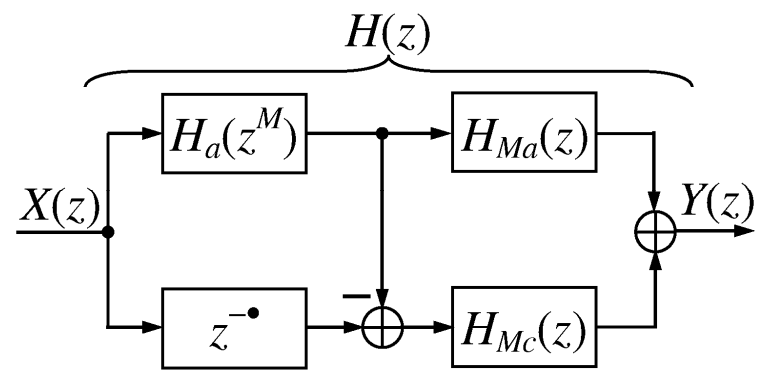

Fig.1 The structure of a filter synthesized using the FRM technique.

Many different optimization techniques have been developed for optimizing the sub-filters of Fig.1. For a given set of frequency response requirements imposed on $H(z)$, there is a wide range of sub-filter frequency responses that can meet the requirement. The coefficient sensitivity of $H(z)$ depend on the frequency responses of the sub-filters and may differ by many orders of magnitudes for different choice of $H_{a}\left(z^{M}\right), H_{M a}(z)$, and $H_{M c}(z)$. Section II shows an example of an FRM-based filter optimized only for overall frequency response performance under infinite precision condition disregarding the coefficient sensitivity. Thus, it is important to steer the optimization algorithm during the course of optimization so as to produce a design with desirable coefficient sensitivity. This paper addresses the issue of designing FRM-based digital filters with minimum coefficient sensitivity.

\section{COEFFICIENT SENSITIVITY OF SUB-FILTERS DESIGNED FOR OPTIMAL FREQUENCY RESPONSE PERFORMANCE}

Many powerful nonlinear optimization techniques have been developed for the design of the sub-filters. These advanced non-linear optimization techniques jointly optimize all the sub-filters for obtaining the optimum overall frequency response. The frequency response of the overall filter obtained using these nonlinear optimization techniques is significantly better than that obtained by optimizing the sub-filters separately using linear optimization technique. Unfortunately, even though the filter designed using these advanced techniques has good overall frequency response under infinite precision arithmetic condition; its coefficient sensitivity may be undesirable; the coefficient values may be very large. We shall illustrate this problem by means of an example.

Consider the design of a low-pass filter with band edges at $0.3 f_{s}$ and $0.305 f_{s}$, respectively, where $f_{s}$ is the sampling frequency. The allowed peak ripple magnitude is 0.01 . When the peak ripple magnitude is used as the objective function for minimization, there are a large number of solutions with almost the same objective function values. The optimization algorithm may converge to any one of the solutions if no further criterion is imposed. The coefficient values for a typical solution are shown in Table I. The value of $M$ in $H_{a}\left(z^{M}\right)$ is 9 . As can be seen from Table I, the coefficients of $H_{a}(z)$ have very large magnitude resulting in serious coefficient sensitivity problem.

\section{COEFFICIENT SENSITIVITY}

The frequency response of the overall filter $H\left(e^{j \omega}\right)$ is given by 
TABLE I

COEFFICIENT VALUES FOR THE EXAMPLE OF SECTION II.

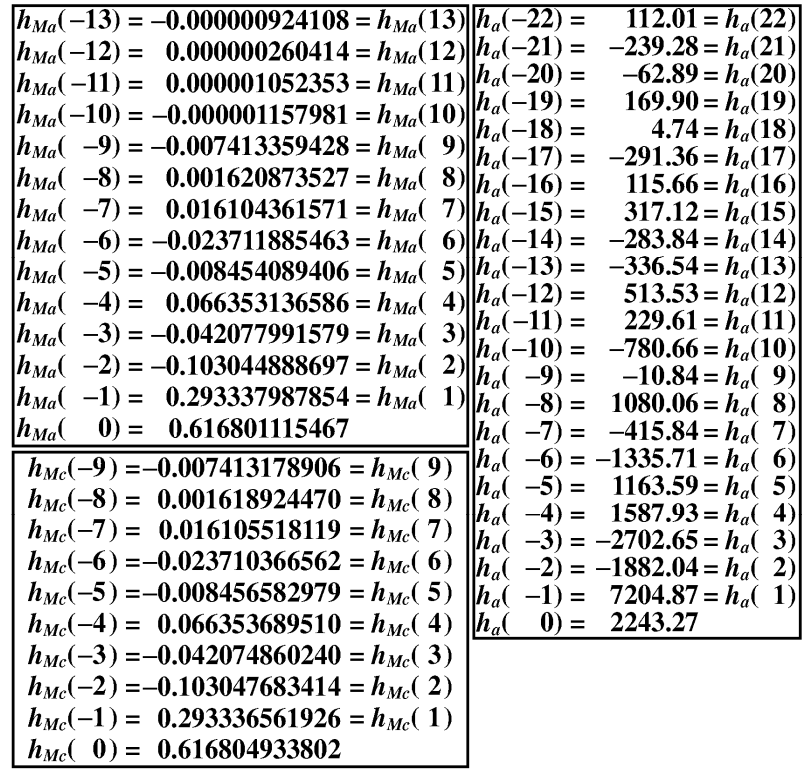

$H\left(e^{j \omega}\right)=H_{a}\left(e^{j \omega M}\right) H_{M a}\left(e^{j \omega}\right)+\left\{e^{-j \omega \bullet}-H_{a}\left(e^{j \omega M}\right)\right\} H_{M c}\left(e^{j \omega}\right)$

Let the $n^{\text {th }}$ coefficient values of $H_{a}\left(z^{M}\right), H_{M a}(z)$, and $H_{M c}(z)$ be $h_{a}(n), h_{M a}(n)$, and $h_{M c}(n)$, respectively. When the coefficient values are made discrete, round off errors are introduced into the coefficient values. Let the errors introduced into $h_{a}(n), h_{M a}(n)$, and $h_{M c}(n)$ be $\Delta h_{a}(n), \Delta h_{M a}(n)$, and $\Delta h_{M c}(n)$, respectively. We shall investigate the change in $H\left(e^{j \omega}\right)$ caused by small values of $\left|\Delta h_{a}(n)\right|,\left|\Delta h_{M a}(n)\right|$, and $\left|\Delta h_{M c}(n)\right|$ where $|x|$ denotes "the magnitude of $x$ ". Suppose that $H\left(e^{j \omega}\right), H_{a}\left(e^{j \omega M}\right), H_{M a}\left(e^{j \omega}\right)$, and $H_{M c}\left(e^{j \omega}\right)$ become $H\left(e^{j \omega}\right)$ $+\Delta H\left(e^{j \omega}\right), H_{a}\left(e^{j \omega M}\right)+\Delta H_{a}\left(e^{j \omega M}\right), H_{M a}\left(e^{j \omega}\right)+\Delta H_{M a}\left(e^{j \omega}\right)$, and $H_{M c}\left(e^{j \omega}\right)+\Delta H_{M c}\left(e^{j \omega}\right)$, respectively, when $h_{a}(n), h_{M a}(n)$, and $h_{M c}(n)$ become $h_{a}(n)+\Delta h_{a}(n), h_{M a}(n)+\Delta h_{M a}(n)$, and $h_{M c}(n)$ $+\Delta h_{M c}(n)$. We shall assume that $\left|\Delta H\left(e^{j \omega}\right)\right|,\left|\Delta H_{a}\left(e^{j \omega M}\right)\right|$, $\left|\Delta H_{M a}\left(e^{j \omega}\right)\right|$, and $\left|\Delta H_{M c}\left(e^{j \omega}\right)\right|$ are small. Thus, neglecting the second order terms, we have

$$
\begin{aligned}
& \Delta H\left(e^{j \omega}\right)=\left\{H_{M a}\left(e^{j \omega}\right)-H_{M c}\left(e^{j \omega}\right)\right\} \Delta H_{a}\left(e^{j \omega M}\right)+ \\
& H_{a}\left(e^{j \omega M}\right) \Delta H_{M a}\left(e^{j \omega}\right)+\left\{e^{-j \omega \bullet}-H_{a}\left(e^{j \omega M}\right)\right\} \Delta H_{M c}\left(e^{j \omega}\right)
\end{aligned}
$$

It can be seen from (2) that the magnitudes of the sensitivities of $H\left(e^{j \omega}\right)$ with respect to changes in $H_{a}\left(e^{j \omega M}\right)$, $H_{M a}\left(e^{j \omega}\right)$, and $H_{M c}\left(e^{j \omega}\right)$ are $\left|H_{M a}\left(e^{j \omega}\right)-H_{M c}\left(e^{j \omega}\right)\right|,\left|H_{a}\left(e^{j \omega M}\right)\right|$, and $\left|e^{-j \omega \bullet}-H_{a}\left(e^{j \omega M}\right)\right|$, respectively. Thus, $\mid H_{M a}\left(e^{j \omega}\right)-$ $H_{M c}\left(e^{j \omega}\right)|, \quad| H_{a}\left(e^{j \omega M}\right) \mid$, and $\left|e^{-j \omega \bullet}-H_{a}\left(e^{j \omega M}\right)\right|$ should be minimized for good robustness against changes in $H_{a}\left(e^{j \omega M}\right)$, $H_{M a}\left(e^{j \omega}\right)$, and $H_{M c}\left(e^{j \omega}\right)$. Squaring both sides of (2) leads to $\left\{\Delta H\left(e^{j \omega}\right)\right\}^{2}=\left\{H_{M a}\left(e^{j \omega}\right)-H_{M c}\left(e^{j \omega}\right)\right\}^{2}\left\{\Delta H_{a}\left(e^{j \omega M}\right)\right\}^{2}$

$$
\begin{aligned}
& +\left(H_{a}\left(e^{j \omega M}\right)\right)^{2}\left\{\Delta H_{M a}\left(e^{j \omega}\right)\right\}^{2}+\left\{e^{-j \omega \bullet}-H_{a}\left(e^{j \omega M}\right)\right\}^{2}\left\{\Delta H_{M c}\left(e^{j \omega}\right)\right\}^{2} \\
& +2\left\{H_{M a}\left(e^{j \omega}\right)-H_{M c}\left(e^{j \omega}\right)\right\} H_{a}\left(e^{j \omega M}\right) \Delta H_{a}\left(e^{j \omega M}\right) \Delta H_{M a}\left(e^{j \omega}\right) \\
& +2\left\{H_{M a}\left(e^{j \omega}\right)-H_{M c}\left(e^{j \omega}\right)\right\}\left\{e^{-j \omega \bullet}-H_{a}\left(e^{j \omega M}\right)\right\} \Delta H_{a}\left(e^{j \omega M}\right) \Delta H_{M c}\left(e^{j}\right. \\
& \omega)+2 H_{a}\left(e^{j \omega M}\right)\left\{e^{-j \omega \bullet}-H_{a}\left(e^{j \omega M}\right)\right\} \Delta H_{M a}\left(e^{j \omega}\right) \Delta H_{M c}\left(e^{j \omega}\right)
\end{aligned}
$$

Let $\mathrm{E}\{x\}$ denotes the expected value of $x$. Taking the expected values for both sides of (3) and taking note that

$$
\begin{aligned}
& \mathrm{E}\left\{\Delta H_{a}\left(e^{j \omega M}\right) \Delta H_{M a}\left(e^{j \omega}\right)\right\}=\mathrm{E}\left\{\Delta H_{a}\left(e^{j \omega M}\right) \Delta H_{M c}\left(e^{j \omega}\right)\right\}= \\
& \mathrm{E}\left\{\Delta H_{M a}\left(e^{j \omega}\right) \Delta H_{M c}\left(e^{j \omega}\right)\right\}=0, \text { we have } \\
& \mathrm{E}\left\{\left(\Delta H\left(e^{j \omega}\right)\right)^{2}\right\}=\left\{H_{M a}\left(e^{j \omega}\right)-H_{M c}\left(e^{j \omega}\right)\right\}^{2} \mathrm{E}\left\{\left(\Delta H_{a}\left(e^{j \omega M}\right)\right)^{2}\right\} \\
&+\left(H_{a}\left(e^{j \omega M}\right)\right)^{2} \mathrm{E}\left\{\left(\Delta H_{M a}\left(e^{j \omega}\right)\right)^{2}\right\} \\
&+\left\{e^{-j \omega \bullet}-H_{a}\left(e^{j \omega M}\right)\right\}^{2} \mathrm{E}\left\{\left(\Delta H_{M c}\left(e^{j \omega}\right)\right)^{2}\right\}
\end{aligned}
$$

Depending on the parity of $N_{x}$, the frequency response $H_{x}\left(e^{j \omega}\right)$ of a symmetrical impulse response FIR filter with length $N_{x}$ and coefficient values $h_{x}(n), n=0, \ldots, N_{x}-1$ is given by either $(5 \mathrm{a})$ or $(5 \mathrm{~b})$

$$
\begin{aligned}
& H_{x}\left(e^{j \omega}\right)=e^{-j \frac{N_{x}-1}{2} \omega} \sum_{n=1}^{N_{x} / 2} 2 h_{x}\left(\frac{N_{x}}{2}-n\right) \cos \left(\omega\left(n-\frac{1}{2}\right)\right. \\
& H_{x}\left(e^{j \omega}\right)=e^{-j \frac{N_{x}-1}{2} \omega}\left\{h_{x}\left(\frac{N_{x}-1}{2}\right)+2 \sum_{n=1}^{\frac{N_{x}-1}{2}} h_{x}\left(\frac{N_{x}-1}{2}-n\right) \cos (\omega n)\right\}
\end{aligned}
$$

Suppose that changing $h_{x}(n)$ to $h_{x}(n)+\Delta h_{x}(n)$ causes $H_{x}\left(e^{j \omega}\right)$ to

change to $H_{x}\left(e^{j \omega}\right)+\Delta H_{x}\left(e^{j \omega}\right)$. Thus, depending on the parity of $N_{x}$, we have either (6a) or (6b)

$$
\begin{gathered}
\Delta H_{x}\left(e^{j \omega}\right)=e^{-j \frac{N_{x}-1}{2} \omega} \sum_{n=1}^{N_{x} / 2} 2 \Delta h_{x}\left(\frac{N_{x}}{2}-n\right) \cos \left(\omega\left(n-\frac{1}{2}\right)\right. \\
\Delta H_{x}\left(e^{j \omega}\right)=e^{-j \frac{N_{x}-1}{2} \omega}\left\{\Delta h_{x}\left(\frac{N_{x}-1}{2}\right)+2 \sum_{n=1}^{\frac{N_{x}-1}{2}} \Delta h_{x}\left(\frac{N_{x}-1}{2}-n\right) \cos (\omega n)\right\}
\end{gathered}
$$

Assume that for $i \neq j$,

$$
\mathrm{E}\left\{\Delta h_{x}(i) \Delta h_{x}(j)\right\}=0
$$

Define the quantity $\varepsilon^{2}$ as

$$
\varepsilon^{2}=\mathrm{E}\left\{\left(\Delta h_{x}(i)\right)^{2}\right\}
$$

Define

$$
\left\|\Delta H_{x}\left(e^{j \omega}\right)\right\|^{2}=\frac{1}{2 \pi} \int_{-\pi}^{\pi} \mathrm{E}\left\{\left(\Delta H_{x}\left(e^{j \omega}\right)\right)^{2}\right\} \mathrm{d} \omega
$$

From (6), (7), (8) and (9), we have

$$
\left\|\Delta H_{x}\left(e^{j \omega}\right)\right\|^{2}=N_{x} \mathrm{E}\left\{\left(\Delta h_{x}(n)\right)^{2}\right\}=N_{x} \varepsilon^{2}
$$

Although $\Delta H_{x}\left(e^{j \omega}\right)$ is a function of $\omega$ for a given filter, $\mathrm{E}\left\{\left(\Delta H_{x}\left(e^{j \omega}\right)\right)^{2}\right\}$ for a large number of independent filters is a constant independent of $\omega$ if $\Delta h_{x}(i)$ has flat spectrum. Thus,

$$
\mathrm{E}\left\{\left(\Delta H_{x}\left(e^{j \omega}\right)\right)^{2}\right\}=N_{x} \varepsilon^{2}
$$

Applying the result of (11), (4) becomes

$$
\begin{aligned}
\mathrm{E}\left\{\left(\Delta H\left(e^{j \omega}\right)\right)^{2}\right\} & =\varepsilon^{2}\left\{N_{a}\left\{H_{M a}\left(e^{j \omega}\right)-H_{M c}\left(e^{j \omega}\right)\right\}^{2}\right. \\
& \left.+N_{M a} H_{a}\left(e^{j \omega M}\right)^{2}+N_{M c}\left\{e^{-j \omega \bullet}-H_{a}\left(e^{j \omega M}\right)\right\}^{2}\right\}
\end{aligned}
$$

where $N_{a}, N_{M a}$, and $N_{M c}$ are the numbers of coefficients of $H_{a}(z), H_{M a}(z)$, and $H_{M c}(z)$, respectively.

We have

$$
\frac{1}{2 \pi} \int_{-\pi}^{\pi}\left(H_{x}\left(e^{j \omega}\right)\right)^{2} \mathrm{~d} \omega=\sum_{n=0}^{N_{x}-1}\left(h_{x}(n)\right)^{2}
$$

Define

$$
\begin{aligned}
& \left\|h_{a}\right\|^{2}=\sum_{n=0}^{N_{a}-1}\left(h_{a}(n)\right)^{2} \\
& \left\|1-h_{a}\right\|^{2}=\left(1-h_{a}\left(\frac{N_{a}-1}{2}\right)\right)^{2}+2 \sum_{n=0}^{\frac{N_{a}-1}{2}}\left(h_{a}(n)\right)^{2}
\end{aligned}
$$




$$
\left\|h_{M a}-h_{M c}\right\|^{2}=\sum_{n=0}^{N_{M a}-1}\left(h_{M a}(n)-h_{M c}(n)\right)^{2}
$$

For $H_{M a}(z)$ and $H_{M c}(z)$ to produce the same phase shifts so that their outputs can be summed correctly, $H_{M a}(z)$ and $H_{M c}(z)$ must have the same order [1], i.e. $N_{M a}=N_{M c}$. From (12), (13), and (14), we have

$\left\|\Delta H\left(e^{j \omega}\right)\right\|^{2}=\left\{N_{a}\left\|h_{M a}-h_{M c}\right\|^{2}+N_{M a}\left\|h_{a}\right\|^{2}+N_{M c}\left\|1-h_{a}\right\|^{2} \varepsilon^{2}(15)\right.$ Let

$$
S^{2}=N_{a}\left\|h_{M a}-h_{M c}\right\|^{2}+N_{M a}\left\|h_{a}\right\|^{2}+N_{M c}\left\|1-h_{a}\right\|^{2}
$$

From (15) and (16), we have

$$
\left\|\Delta H\left(e^{j \omega}\right)\right\|^{2}=S^{2} \varepsilon^{2}
$$

From (17), and since $\varepsilon^{2}=\mathrm{E}\left\{\left(\Delta h_{x}(i)\right)^{2}\right\}$ by definition, it is clear

that $S^{2}$ is a coefficient sensitivity measure. In order to minimize $S^{2}$, the peak ripple magnitude of $H\left(e^{j \omega}\right)$, denoted as $\delta$, may be set as a constraint and $S^{2}$ becomes the objective for minimization as in (18).

Minimize $S^{2}=N_{a}\left\|h_{M a}-h_{M c}\right\|^{2}+N_{M a}\left\|h_{a}\right\|^{2}+N_{M c}\left\|1-h_{a}\right\|^{2}(18 \mathrm{a})$ subject to $\delta \leq \delta_{0}$

In (18b), $\delta_{0}$ is a predefined constant.

\section{A MINIMUM SENSITIVITY EXAMPLE}

We choose the design of a low-pass filter with the same band edges, $M$ value for $H_{a}\left(z^{M}\right)$, and sub-filter lengths as the filter shown in Section II as an example to illustrate the superiority of this new design technique. The peak ripple magnitude is relaxed by $2 \%$ to 0.0102 and (18a) is used as the objective function. The coefficient values of this new

\section{TABLE II}

COEFFICIENT VALUES FOR $H_{a}(z), H_{M a}(z)$, AND $H_{M c}(z)$ FOR THE FILTER OBTAINED BY MINIMIZING $S^{2}$.

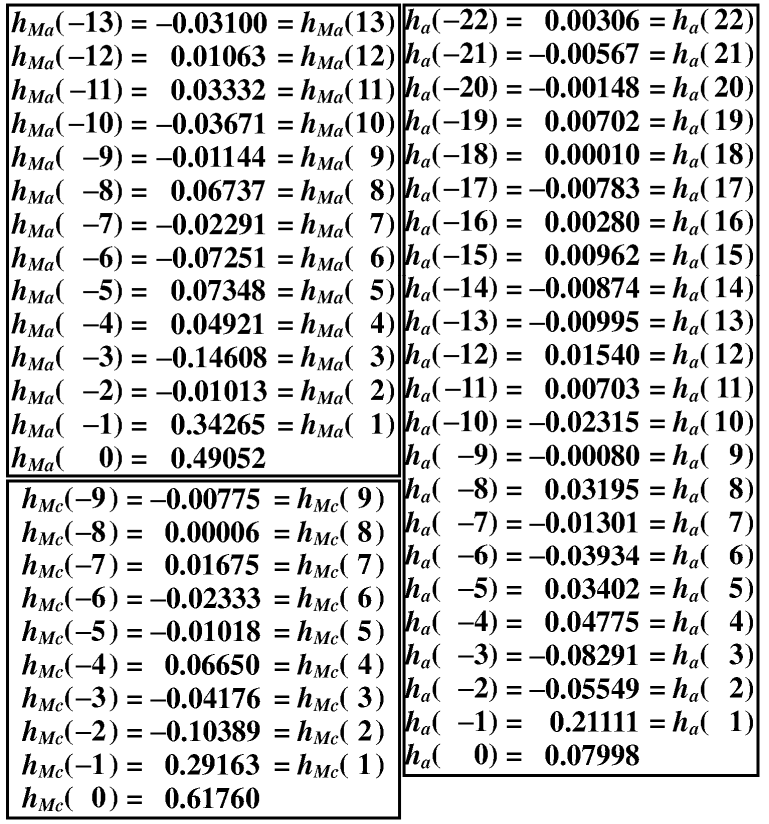

design are shown in Table II. The value of $S^{2}$ is 26.43 and the peak ripple magnitudes of the overall filter become
0.01029 if the coefficient quantization step sizes for each coefficient in Table II are $2^{-14}$.

For the purpose of comparison, the value of $N_{a} \| h_{M a}-$ $h_{M c}\left\|^{2}+N_{M a}\right\| h_{a}\left\|^{2}+N_{M c}\right\| 1-h_{a} \|^{2}$ (i.e. the equivalent $S^{2}$ value) for the filter whose coefficients are shown in Table I is $6.78 \times 10^{9}$. High coefficient sensitivity is expected. To achieve a peak ripple magnitude of about 0.0103 the coefficient quantization step size for the filter shown in Table I should not be larger than $2^{-27}$.

\section{CONCLUSIONS}

In this paper, we present a technique that includes coefficient sensitivity measure into the objective function of the optimization algorithm used for designing FRM based FIR filters. It results in filters with low coefficient sensitivity.

\section{REFERENCES}

[1] Y. C. Lim, "Frequency-Response Masking Approach for the Synthesis of Sharp Linear Phase Digital Filter," IEEE Transactions on Circuits and Systems, vol. CAS-33, pp.357-364, April 1986.

[2] G. Rajan, Y. Neuvo, and S.K. Mitra, "On the design of sharp cutoff wideband fir filters with reduced arithmetic complexity," IEEE Transactions on Circuits and Systems, vol- 35, pp.1447-1454, Nov. 1988.

[3] Y. C. Lim and Y. Lian, "The Optimum Design of One- and TwoDimensional FIR Filters Using the Frequency Response Masking Technique," IEEE Transactions on Circuits and Systems II, vol. CAS-40, pp.88-95, February 1993.

[4] J.H. Lee and C.K. Chen, "Design of sharp FIR filters with prescribed group delay," in Proc. IEEE International Symposium on Circuits and Systems, vol.1, pp. 92-95, 1993.

[5] Y. C. Lim and Y. Lian, "Frequency-response masking approach for digital filter design: complexity reduction via masking filter factorization," IEEE Transactions on Circuits and Systems II, vol. CAS-II-41, no. 8, pp. 518-525, August 1994.

[6] M. G. Bellanger, "Improved Design of Long FIR Filters Using the Frequency Masking Technique," in Proc. IEEE Int. Conf. Acoust. Speech. Signal Processing, pp.1272-1275, 1996.

[7] L. C. R. Barcellos, S. L. Netto, and P. S. R. Diniz, "Optimization of FRM filters using the WLS-Chebyshev approach," Circuits, Systems, and Signal Processing, vol. 22, no.2, pp. 99-113, March/April 2003.

[8] T. Saramäki, and Y. C. Lim, "Use of the Remez algorithm for designing FRM based FIR filters," Circuits, Systems, and Signal Processing, vol. 22, no.2, pp.77-97, March/April 2003.

[9] W. S. Lu and T. Hinamoto, "Optimal Design of FrequencyResponse-Masking Filters Using Semidefinite Programming," IEEE Transactions on Circuits and Systems I, vol. 50, pp.557-568, no. 4, April 2003

[10] Y. Lian, and C. Z. Yang, "Complexity reduction by decoupling the masking filters from bandedge shaping filter in FRM technique," Circuits, Systems, and Signal Processing, vol. 22, no.2, pp.115-135, March/April 2003.

[11] Y. Lian, "Complexity reduction for FRM based FIR filters using the prefilter-equalizer technique," Circuits, Systems, and Signal Processing, vol. 22, no.2, pp.137-155, March/April 2003.

[12] T. Saramäki, J. Yli-Kaakinen, and H. Johansson, "Optimization of Frequency-Response-Masking Based FIR Filters," Circuits, Systems, and Computers, vol. 12, no. 5, pp. 563-590, October 2003.

[13] W. R. Lee, V. Rehbock, and K. L. Teo, "Frequency-Response Masking Based FIR Filter Design with Power-of-Two Coefficients and Suboptimum PWR," Circuits, Systems, and Computers, vol. 12, no. 5, pp. 591-600, October 2003.

[14] O. Gustafsson, H. Johansson, and L. Wanhammar, "Single Filter Frequency-Response Masking FIR Filter," Circuits, Systems, and Computers, vol. 12, no. 5, pp. 601-630, October 2003. 
[15] W. S. Lu and T. Hinamoto, "Optimal design of IIR FrequencyResponse- Masking filters Using Second-Order Cone Programming," IEEE Transactions on Circuits and Systems I, vol. 50, pp.1401-112, no. 11, Nov. 2003.

[16] W.R. Lee, V. Rehbock, K.L. Teo, and L. Caccetta, "A weighted least-square-based approach to FIR filter design using the frequencyresponse masking technique," IEEE Signal Processing Letters, vol11, pp. 593-596, Jul 2004.

[17] A. Usman and S.A. Khan "Simulation of frequency response masking approach for FIR filter design," WSEAS Transactions on Systems, vol-3, no-10, pp.2919-24, Dec. 2004.

[18] Y. J. Yu, K. L. Teo, Y. C. Lim, and G. H. Zhao, "Extrapolated Impulse Response Filter and its Application in the Synthesis of Digital Filters Using the Frequency-Response Masking Technique", Signal Processing (Elsevier), Vol 85/3, pp. 581-590, March 2005.

[19] J.X. Rodrigues and K.R. Pai, "Modified linear phase frequency response masking FIR filter," Proc. 4th International Symposium on Image and Signal Processing and Analysis, p 434-439, Sep. 2005.

[20] L. Cen and Y. Lian "Hybrid Genetic Algorithm for the Design of Modified Frequency-Response Masking Filters in a Discrete Space" Circuits, Systems, and Signal Processing, vol. 25, no.2, pp 153-174, April 2006.

[21] T. Saramäki, Y. C. Lim, and R. Yang, "The Synthesis of Half-Band Filter Using Frequency-Response Masking Technique," IEEE Transactions on Circuits and Systems II, vol.42, pp.58-60, January 1995.

[22] Y. Lian, "The optimum design of half-band filter using multi-stage frequency-response masking technique, " Signal Processing (Elsevier Science), vol-44, no.3, pp. 369-372, July 1995.

[23] C. T. Thia and Y. Lian, "The implementation of a high-speed 645-tap equivalent half-band FIR filter using the frequency response masking technique," Proc. IEEE TENCON 2004, vol-1, pp.13-16, Nov. 2004.

[24] Y. C. Lim and S. H. Low, "Frequency-response masking approach for the synthesis of sharp Two-dimensional diamond-shaped filters," IEEE Transactions on Circuits and Systems II, vol. CAS-II-45, no. 12, pp. 1573-1584, December 1998.

[25] H. Johansson and L. Wanhammar, "High-speed recursive digital filters based on the frequency-response masking approach," IEEE Transactions on Circuits and Systems II, vol. 47, pp. 48-61, Jan. 2000.

[26] M.D. Lutovac and L.D Milic, "IIR filters based on frequencyresponse masking approach," in Proc. 5th International Conference on Telecommunications in Modern Satellite, Cable and Broadcasting Service, vol.1, pt. 1, pp 163-70, 2001

[27] O. Gustafsson, H. Johansson, and L. Wanhammar, "Single filter frequency masking high-speed recursive digital filters," Circuits, Systems, and Signal Processing, vol. 22, no.2, pp.219-238, March/April 2003.

[28] H.H. Chen, S.C. Chan, and K.L. Ho, "A semi-definite programming (SDP) method for designing IIR sharp cut-off digital filters using frequency-response masking," in Proc. IEEE International Symposium on Circuits and Systems, vol-3, pp III 149-152, 2004.

[29] J. W. Lee and Y. C. Lim, "Efficient implementation of real filter banks using frequency response masking techniques," in Proceedings of the 2002 IEEE Asia Pacific Conference on Circuits and Systems, vol-1, pp.69-72. Indonesia.

[30] L. Rosenbaum, P. Lowenborg, and H. Johansson, "Cosine and sine modulated FIR filter banks utilizing the frequency-response masking approach," in Proc. IEEE International Symposium on Circuits and Systems, vol-3, pp. III 882-885, 2003.

[31] H. Johansson and T. Saramäki, "Two-channel FIR filter banks utilizing the FRM approach," Circuits, Systems, and Signal Processing, vol. 22, no.2, pp.157-192, March/April 2003.

[32] M. B. Furtado, P. S. R. Diniz, and S. L. Netto, "Optimized prototype filter based on the FRM approach for cosine-modulated filter banks," Circuits, Systems, and Signal Processing, vol. 22, no.2, pp. 193-210, March/April 2003.

[33] S. L. Netto, L. C. R. Barcellos, and P. S. R. Diniz, "Efficient Design of Narrowband Cosine-Modulated Filter Banks Using a Two-Stage Frequency-Response Masking Approach," Circuits, Systems, and Computers, vol. 12, no. 5, pp. 631-642, October 2003.

[34] R. Bregovic and T. Saramaki, "Design of two-channels FIR filterbanks with rational sampling factors using the FRM technique," in Proc. IEEE International Symposium on Circuits and Systems, vol-2, pp.1098-1101, May 2005.
[35] Y.C. Lim and R. Yang, "On the Synthesis of Very Sharp Decimators and Interpolators Using the Frequency-Response Masking Technique," IEEE Transactions on Signal Processing, vol. SP-53, no.4, pp. 1387-1397, April 2005.

[36] Hakan Johansson "Two Classes of Frequency-Response Masking Linear-Phase FIR Filters for Interpolation and Decimation," Circuits, Systems, and Signal Processing, vol. 25, no.2, pp 175-200, April 2006.

[37] Y. C. Lim and Y. J. Yu, "Synthesis of Very Sharp Hilbert Transformer Using the Frequency-Response Masking Technique," IEEE Transactions on Signal Processing, vol. SP-53, pp. 2595-2597, July 2005.

[38] Y. C. Lim, Y.J. Yu, and T. Saramaki, "Optimum Masking Levels and Coefficient Sparseness for Hilbert Transformers and Half-Band Filters Designed Using the Frequency-Response Masking Technique," IEEE Transactions on Circuits and Systems I, vol. CASI-52, no.11, pp. 2444-2453, November 2005.

[39] Y. C. Lim, Y. J. Yu, H. Q. Zheng, and S. W. Foo, "FPGA Implementation of digital filters synthesized using the FRM technique," Circuits, Systems, and Signal Processing, vol. 22, no.2, pp.211-218, March/April 2003.

[40] Y. Lian, "A Modified Frequency Response Masking Structure for High-Speed FPGA Implementation of Programmable Sharp FIR Filters," Circuits, Systems, and Computers, vol. 12, no. 5, pp. 643654, October 2003

[41] W. Boonkumklao, Y. Miyanaga, K. Dejhan, "A flexible architecture for digital signal processing," IEICE Transactions on Information and Systems, Pt. E86D, pp. 2179-2186, Oct. 2003.

[42] Paulo S. R. Diniz, Luiz C. R. de Barcellos, and Sergio L. Netto, "Design of High-Resolution Cosine-Modulated Transmultiplexers with Sharp Transition Band," IEEE Transactions on Signal Processing, vol.52, pp. 1278-1288, May 2004.

[43] Y. Lian and J.H. Yu, "The reduction of noises in ECG signal using a frequency response masking based FIR filter," Proc. 2004 IEEE International Workshop on Biomedical Circuits and Systems, pp. S2/4 17-20, 2004

[44] Y. Lian and Y Wei, "A computationally efficient nonuniform FIR digital filter bank for hearing aids," IEEE Transactions on Circuits and Systems I: Fundamental Theory and Applications, vol.-52, no-12, pp 2754-62, Dec. 2005.

[45] Lim Y C, "A digital filter bank for digital audio systems," IEEE Transactions on Circuits and Systems, vol. CAS-33, no. 8, pp. 848849, August 1986.

[46] R.H. Yang, S.B. Chiah, and W.Y. Chan, "Design and implementation of a digital audio tone control unit using an efficient FIR filter structure," Proc. IEEE Region 10 Annual International Conference, vol-1, pp. 273-277, 1996.

[47] C.S. Lin and C. Kyriakakis, "Frequency response masking approach for designing filter banks with rational sampling factors," in Proc. 2003 IEEE Workshop on Applications of Signal Processing to Audio and Acoustics, pp 99-102, Oct 2003.

[48] S. W. Foo and W. T. Lee, "Application of Fast Filter Bank for Transcription of Polyphonic Signals," Circuits, Systems, and Computers, vol. 12, no. 5, pp. 654-674, October 2003.

[49] S.W. Foo and W.T. Lee, "Recognition of piano notes with the aid of FRM filters," in Proc. International Symposium on Control, Communications and Signal Processing, pp. 409-413, 2004

[50] N. Hayasaka, N. Wada, S. Yoshizawa, and Y. Miyanaga, "A robust speech recognition system using FRM running spectrum filtering," Proc. International Symposium on Control, Communications and Signal Processing, pp. 401-404, 2004.

[51] Y. Liu and Z. Lin "On the Applications of the Frequency-Response Masking Technique in Array Beamforming," Circuits, Systems, and Signal Processing, vol. 25, no.2, pp 201-224, April 2006.

[52] Y. Zhang; W. Wu, and B. Tian, "A novel sharp-cutoff FIR filter design technique and its application in software radio," Proc. International Conference on Communication Technology, Vol-2, pp. 1821 - 18259, April 2003.

[53] H. Saleh, E. Zimmermann, G. Brandenburg, and H. Halling, "Efficient FPGA-based multistage two-path decimation filter for noise thermometer," Proc. The 13th International Conference on Microelectronics, pp. 161- 164, Oct 2001. 